potents in each semigroup was a finite discrete set. It might be of interest to know if there exists a semigroup $S=E S E$ which is compact connected, has a zero, is not acyclic and such that the set of idempotents is connected.

\title{
BIBLIOGRAPHY
}

1. A. D. Wallace, Acyclicity of compact connected semigroups, Fund. Math. 1 (1961), 99-105.

2. - Cohomology, dimension and mobs, Summa Brasil. Math. 3 (1953), 43-55.

3. - - Problems on semigroups, Colloq. Math. 8 (1961), 223-224.

TUlane University

\section{CONFORMAL VECTOR FIELDS IN COMPACT RIEMANNIAN MANIFOLDS}

T. K. PAN

1. Introduction. Let $V^{n}$ be a compact Riemannian manifold of dimension $n$ and of class $C^{3}$. Let $g_{i j}(x)$ of class $C^{2}$ be the coefficients of the fundamental metric which is assumed to be positive definite. Let $\Gamma_{i j}^{h}$ be the Christoffell symbol, $R_{i j h k}$ the curvature tensor and $R_{i j}$ the Ricci tensor.

Let $\phi$ be an arbitrary scalar invariant, $\xi^{i}$ an arbitrary vector field and $\xi_{i_{1} i_{2}} \ldots i_{p}$ an arbitrary anti-symmetric tensor field of order $p$, all of class $C^{2}$ in $V^{n}$. We shall make use of the following results obtained by S. Bochner and K. Yano [1, pp. 31, 51, 69]:

(1.1) $\left(\Delta \phi \geqq 0\right.$ everywhere in $\left.V^{n}\right) \Rightarrow\left(\phi=\right.$ constant everywhere in $\left.V^{n}\right)$.

$$
\int_{V^{n}} \xi^{i}, i d v=0
$$

$$
\begin{gathered}
\int_{V^{n}}\left(R_{i j} \xi^{i} \xi^{j}+\xi^{i},_{j} \xi^{j},{ }_{i}-\xi^{i},{ }_{i} \xi^{j},{ }_{j}\right) d v=0 . \\
\int_{V^{n}}\left(F\left\{\xi_{i_{1} i_{2} \cdots i_{p}}\right\}+\xi^{i i_{2} \cdots i_{p}, j} \xi_{j i_{2} \cdots i_{p}, i}-\xi^{i i_{2} \cdots i_{p},{ }_{i} \xi^{j}{ }_{i_{2}} \cdots i_{p}, j}\right) d v=0
\end{gathered}
$$

where

Presented to the Society, August 29, 1961; received by the editors January 2, 1962 and, in revised form, May 10, 1962. 


$$
F\left\{\xi_{i_{1} i_{2} \cdots i_{p}}\right\}=R_{i j} \xi^{i i_{2} \cdots i_{p}} \xi_{i_{2} \cdots i_{p}}^{i_{1}}+\frac{p-1}{2} R_{i j k l} \xi^{i j i_{3} \cdots i_{p}} \xi_{i_{3} \cdots i_{p}}^{k l}
$$

and $V^{n}$ in equations (1.2) to (1.4) is assumed to be orientable.

If a vector field $\xi^{i}$ defines a one-parameter continuous group of infinitesimal conformal transformations in $V^{n}$, the intrinsic derivative of $\xi_{i} d x^{i} / d s$ along any geodesic $x^{i}(s)$ depends only on the point and not on the direction of the geodesic passing through the point; that is

$$
\xi_{i, j} \frac{d x^{i}}{d s} \frac{d x^{j}}{d s}=\frac{1}{n} \xi^{i},{ }_{i} .
$$

Let $\xi^{i}$ be an arbitrary vector field which does not necessarily define a one-parameter continuous group of infinitesimal conformal transformations. Then, instead of (1.5), we have in general

$$
\xi_{i, j} \frac{d x^{i}}{d s} \frac{d x^{j}}{d s}=f\left(x^{i}, \xi^{i}, \frac{d x^{i}}{d s}\right) .
$$

In the following, such $\xi^{i}$ will be associated with an arbitrary but fixed scalar field $\phi$ in a certain way to give a generalization of some wellknown vector fields.

2. Definition. Let $\lambda^{i}$ be an arbitrary unit vector field. Consider $M$ defined by

$$
M=\xi_{i, j} \lambda^{i} \lambda^{j}-\phi
$$

When $\xi^{i}$ defines a one-parameter continuous group of infinitesimal conformal transformations and $\phi=\xi^{i}, i / n$, we have, by (1.5), $M=0$. Hence it seems appropriate to call $M$ the $\phi$-conformal measure of $\xi^{i}$ with respect to $\lambda^{i}$.

Let $\lambda_{\alpha} \mid{ }^{i}, \alpha=1,2, \cdots, n$, be $n$ mutually orthogonal unit vector fields in $V^{n}$ and $M_{\alpha}$ the $\phi$-conformal measures of $\xi^{i}$ with respect to them, that is

$$
M_{\alpha}=\left.\left.\xi_{i, j} \lambda_{\alpha}\right|^{i} \lambda_{\alpha}\right|^{j}-\phi .
$$

The mean of $M_{\alpha}$ is equal to

$$
\frac{1}{n} \sum_{\alpha=1}^{n} M_{\alpha}=\frac{1}{n} g^{i j} \xi_{i, j}-\phi
$$

which is evidently independent of the choice of the orthogonal ennuple $\left.\lambda_{\alpha}\right|^{i}$. A vector field $\xi^{i}$ for which the mean (2.2) vanishes is 
called a $\phi$-conformal vector field in $V^{n}$ or simply a conformal vector field in $V^{n}$. To each $\phi$ there corresponds one conformal vector field. The definition leads immediately to the following necessary and sufficient condition

$$
\xi^{i},{ }_{i}=n \phi .
$$

Since a harmonic vector field and a Killing vector field satisfy (2.3) when $\phi=0$, a conformal vector field may be considered as a generalization of them in this sense.

We intend to investigate properties of conformal vector fields in a compact Riemannian manifold $V^{n}$, particularly the global nonexistence of these fields in $V^{n}$. A generalization of our concept to tensor fields is given at the end of the paper.

3. Properties. By definition of the Laplacean (denoted by $\Delta$ ) of a scalar field, we obtain for a conformal vector field $\xi^{i}$

$$
\Delta \xi^{i},_{i}=g^{j k} \xi^{i},_{i j k}=n \Delta \phi .
$$

The tendency of a vector $v^{i}$ in a unit direction $a^{i}$ is defined as the projection of the vector $a^{k} v^{i}{ }_{, k}$ in the direction of $a^{i}$. It is well known that the divergence of a vector in $V^{n}$ is the sum of tendencies of the vector for $n$ mutually orthogonal directions in $V^{n}$ and that of a unit vector in $V^{2}$ is numerically equal to the geodesic curvature of a curve normal to the vector $[2$, p. $422 ; 3$, p. 201$]$. Hence from (1.1) and (1.2) we have the following two theorems.

THEOREM 3.1. If $\phi$ satisfies $\Delta \phi \geqq 0$ everywhere in a compact Riemannian manifold $V^{n}$, then the sum of tendencies of the $\phi$-conformal vector field for $n$ mutually orthogonal directions is constant throughout the manifold. If $\phi$ satisfies $\Delta \phi \geqq 0$ everywhere in a compact Riemannian manifold $V^{2}$, then the geodesic curvature of the orthogonal trajectories of the curves of the $\phi$-conformal unit vector field is constant throughout the manifold.

THEOREM 3.2. In a compact orientable Riemannian manifold $V^{n}$, there exists no $\phi$-conformal vector field with $\phi>0$ or $\phi<0$ everywhere in the manifold and therefore the divergence of a $\phi$-conformal vector field is a constant everywhere in $V^{n}$ if and only if the constant is zero. In a compact orientable Riemannian manifold $V^{2}$, the orthogonal trajectories of the curves of a $\phi$-conformal unit vector field form a family of geodesics in the manifold if and only if the divergence of the vector field is constant throughout.

By (2.3) we may write (1.3) as 


$$
\int_{V^{n}}\left(\xi^{i},{ }_{j} \xi^{j},_{i}+R_{i j} \xi^{i} \xi^{j}\right) d v=\int_{V^{n}} n^{2} \phi^{2} d v
$$

and

$$
\int_{V^{n}}\left(\xi^{i}, \xi^{j},_{i}-n^{2} \phi^{2}\right) d v=-\int_{V^{n}} R_{i j} \xi^{i} \xi^{i} d v
$$

which lead to the following two theorems:

THEOREM 3.3. In a compact orientable Riemannian manifold $V^{n}$ there exists no $\phi$-conformal vector field $\xi^{i}$ which satisfies

$$
\int_{V^{n}}\left(R_{i j} \xi^{i} \xi^{j}+\xi^{i},{ }_{j} \xi^{j},{ }_{i}\right) d v \leqq 0
$$

unless we have $\phi=0$ and then automatically the equality sign holds.

THEOREM 3.4. In a compact orientable Riemannian manifold with negative (positive) definite Ricci curvature throughout, there exists no $\phi$-conformal vector field other than zero vector field which satisfies

$$
\int_{V^{n}}\left(\xi^{i},{ }_{j} \xi^{j},{ }_{i}-n^{2} \phi^{2}\right) d v \leqq 0 \quad(\geqq 0) .
$$

The above theorem includes as special cases some results about global nonexistence of harmonic vector field and Killing vector field obtained by S. Bochner [1, pp. 37, 39].

4. Generalization. An anti-symmetric tensor field $\xi_{i_{1} i_{2}} \cdots i_{p}$ of order $p$ is a conformal Killing tensor field if and only if

$$
\xi_{i i_{2} \ldots i_{p}, j} \frac{d x^{i}}{d s} \frac{d x^{j}}{d s}=\phi_{i_{2} \cdots i_{p}}
$$

where

$$
\phi_{i_{2} \ldots i_{p}}=\frac{1}{n} g^{i j \xi_{i i_{2}} \ldots i_{p}, j}
$$

is an anti-symmetric tensor of order $p-1[1$, p. 73].

Denote by $\left.\lambda_{\alpha}\right|^{i} n$ mutually orthogonal unit vector fields, by $\phi_{i_{2} i_{3}} \ldots i_{p}$ an arbitrary but fixed anti-symmetric tensor field of order $p-1$, and by $\left.M_{\alpha}\right|_{i_{2} \ldots i_{p}}$ the following tensor fields

$$
\left.M_{\alpha}\right|_{i_{2} \cdots i_{p}}=\xi_{i i_{2}} \cdots i_{p},\left.\left.j \lambda_{\alpha}\right|^{i} \lambda_{\alpha}\right|^{j}-\phi_{i_{2} \cdots i_{p}} .
$$

The mean of $(4.2)$ is equal to 


$$
\left.\frac{1}{n} \sum_{\alpha=1}^{n} M_{\alpha}\right|_{i_{2} \cdots i_{p}}=\frac{1}{n} \xi_{i i_{2} \ldots i_{p}, j} g^{i j}-\phi_{i_{2} \ldots i_{p}}
$$

which is independent of the choice of the orthogonal ennuple $\lambda_{\alpha} \mid$ : An anti-symmetric tensor field $\xi_{i_{1}} \ldots i_{p}$ for which (4.3) vanishes is called a $\phi$-conformal tensor field in $V^{n}$. Thus $\xi_{i_{1}} \cdots_{i_{p}}$ is a $\phi$-conformal tensor field in $V^{n}$ if and only if

$$
g^{i j} \xi_{i i_{2}} \cdots i_{p}, j=n \phi_{i_{2}} \ldots i_{p} \text {. }
$$

Obviously, a $\phi$-conformal tensor field includes a conformal Killing tensor field as a special case.

Substituting (4.4) into (1.4) gives

THEOREM 4.1. In a compact orientable Riemannian manifold there exists no $\phi$-conformal tensor field of order $p$ which satisfies

$$
F\left\{\xi_{i_{1} \cdots i_{p}}\right\}+\xi^{i i_{2} \cdots i_{p}, j} \xi_{j i_{2} \cdots i_{p}, i} \leqq 0
$$

unless we have $\phi_{i_{2}} \cdots i_{p}=0$ and then automatically the equality sign holds.

\section{REFERENCES}

1. K. Yano and S. Bochner, Curvature and Betti numbers, Princeton Univ. Press, Princeton, N. J., 1953.

2. C. E. Weatherburn, Some theorems in Riemannian geometry, Tôhoku Math. J. 38 (1933), 422-430.

3. L. P. Eisenhart, An introduction to differential geometry with the use of the tensor calculus, Princeton Univ. Press, Princeton, N. J., 1947.

UNIVERSITY OF OKLAHOMA 